\title{
An Unsupervised Change Detection in Satellite IMAGES Using MRFFCM Clustering
}

\author{
S.Venkata Lakshmi \\ Dept. of CSE \\ Panimalar Institute of Technology \\ Chennai,India
}

\author{
K.Sathyamoorthy \\ Dept. of CSE \\ Panimalar Institute of Technology \\ Chennai,India
}

\author{
T.K Senthil Kumar \\ Dept. of ECE \\ Rajalakshmi Institute of \\ Technology \\ Chennai,India
}

\begin{abstract}
This paper presents a new approach for change detection in synthetic aperture radar images by incorporating Markov random field (MRF) within the framework of FCM. The objective is to partition the difference image which is generated from multitemporal satellite images into changed and unchanged regions. The difference image is generated from log ratio and mean ratio images by image fusion technique. The quality of difference image depends on image fusion technique. In the present work; we have proposed an image fusion method based on stationary wavelet transform. To process the difference image is to discriminate changed regions from unchanged regions using fuzzy clustering algorithms. The analysis of the DI is done using Markov random field (MRF) approach that exploits the interpixel class dependency in the spatial domain to improve the accuracy of the final change-detection areas. The experimental results on real synthetic aperture radar images demonstrate that change detection results obtained by the MRFFCM exhibits less error than previous approaches. The goodness of the proposed fusion algorithm by well-known image fusion measures and the percentage correct classifications are calculated and verified.
\end{abstract}

Keywords-image change detection, MMRM, LRM, MRFFCM, SAR, fuzzy clustering

\section{INTRODUCTION}

Satellite images and geographic mapping is the key to many applications and it depends on the accurate information about the land area in a region.Upon detecting changes in the geographic area at different times is important in many domains. The images are generated by synthetic aperture radar (SAR)[1][2].The unsupervised[11] change detection approach is classified in to 3 steps.1)preprocessing 2) difference image generation 3)analysis of difference image. Usually satellite images are subject to different errors. The geometric errors are systematic, which means that they can be identified and corrected. The images affected by external errors ie.due to movement of air or spacecraft also to be noticed and corrected.The purpose of internal errors correction is removal of geometrics in images.

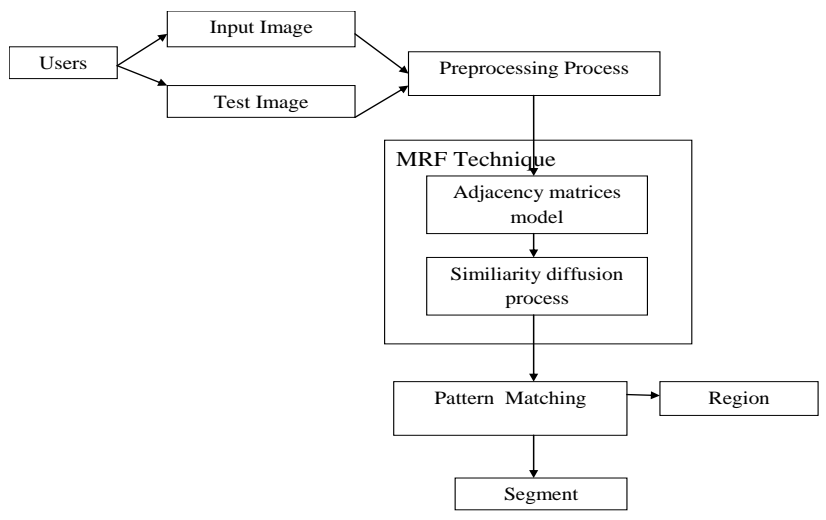

Figure.1. Process of change detection
The noise removal [5] in images is the next process and of most of the SAR images suffer from the presence of speckle noise [4]. The Markov random field is a granular noise that inherently exists in and degrades the quality of SAR images. The next step is to identify the change by comparing two images by pixel by pixel and a new image is generated which is the difference image.

The figure 1 shows the process of the image with MRF technique and the discrimination of changed and unchanged regions are separated using fuzzy clustering algorithms. A mean ratio and log ratio operator is used to identify and by improving MRFFCM clustering algorithms to identify the change areas.The MRFFCM (Markov Random Field Fuzzy C-Means clustering)[4] algorithm is proposed to focus on modification of membership to reduce the effect of speckle noise. The information provided by neighbor pixel serve as the spatial context and it is considered to center pixel in FCM progress. The point wise prior probability is of positive correlation to the new membership, which indicates that we are able to modify the new membership by modifying the point wise prior probability indirectly. This approach is time consuming. The analysis shows that the new approach not only does well in reducing speckle noise but also reduce time complexity.

\section{RELATED WORK}

An approach for sub pixel change detection in satellite imagery[6], detects the land cover changes using remote sensed images. It provides fine information dedicated to a specific range of application and its characteristics result in between fine spatial resolution and fine spectral resolution at high time frequency .Related another work 
represents the changes in satellite imagery by incorporating image fusion and soft clustering techniques. Similar work is analyzed in multitemporal remote sensing images using neural based algorithm

\subsection{DETECTION OF CLUSTER CHANGE:}

In general, Unsupervised Change detection algorithms usually take two digitized images as input and return the locations where the differences between the two images can be identified. To accomplish this task,preprocessing is necessary focused at rendering two images with respect to spatial and spectral domains.Concerning the spatial domain, the coregistered two images are verified whether they are at same coordinates in the images. The crucial step,if performed in accuracy may lead to registration of noise .Illumination can be assumed to change smoothly with respect to pixel coordinates. Therefore in many situations,we can divide an original scene into different areas of interest (AOI),so that differences in illuminance conditions will be constant for each pixel in a given specified area of interest. It is worth-noting that selecting the AOI dimensions should involve a trade-off between obtaining uniform illumination conditions and relying on sufficient statistics for carrying out the change detection process.

The two registered and corrected images (a linear or non linear) combinations of the spectral bands of such images are compared pixel by pixel in order to generate a further image called as "difference image". The difference images is computed in such a way that pixels are associated with land cover changes present graylevel values significantly different from those of pixels associated with unchanged areas. Toexploit the spatial -contextual information[5], input patterns are generated corresponding to each pixel in the difference image, DI considering its spatial neighborhood system as follows.

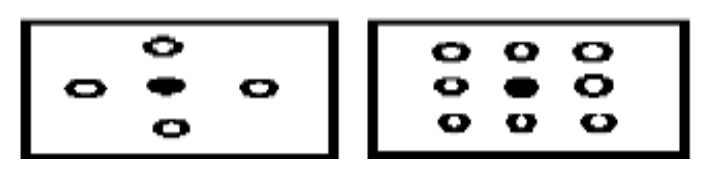

Figure. 2.a) 1st neighborhood b) 2nd neighborhood

Now after,generating a two dimensional pattern corresponding to the pixel at position $(m, n)$ of DI by considering the gray value of pixels as one feature and average of the gray value of 8 neighbors as another feature .However when SAR images are considered, changes are obtained by analyzing the image resulting from the applications of the ratio operator to the considered couple of SAR images with mean and log ratios.Hence image fusion technique is introduced to generate a difference image by using complementary information from a mean ratio image and log ratio image. Image fusion is a process of fusing two or more images into a single fused image, there by relevant information in the images are combined. . So this single fused image will be more informative than any of the input images.

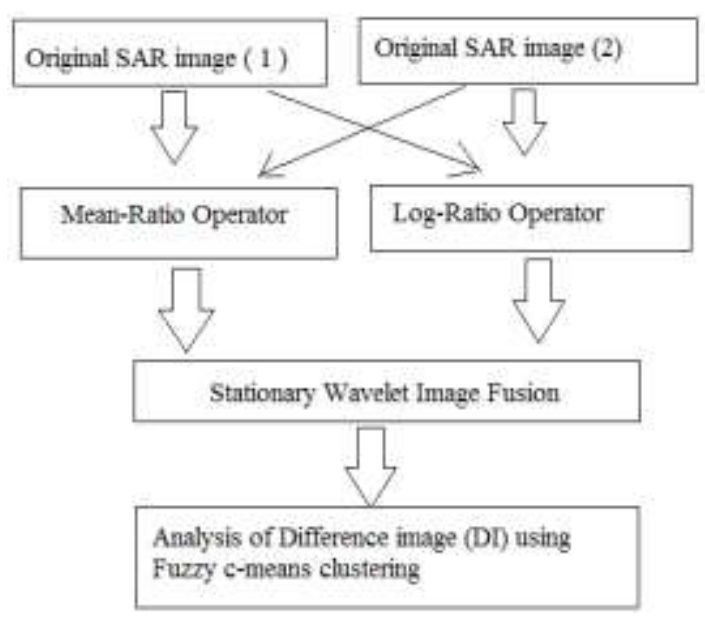

Figure 3: Process of Image Fusion based on the Stationary Wavelet Transform

In the newly produced difference image, the pixel value of no change area approach zero and the pixel value of changed area are positive or negative .The majority of fusion technique are based on wavelet transformation but the image fusion is resulting with shift variant and additive noise in fused image and it doesn't preserve edge a number of clusters of the image. The information loss is more thereby, the clarity of the fused image is also reduced .Ground truth image is finally obtained in which a pixel on the SAR image is compared to what is there in reality (at the present time) in order to verify the contents of the image. It helps to determine the accuracy and thereby minimize errors in the classification.

\subsection{Cluster change analysis using Minimum Mean Ratio Method (MMRM):}

The MMRM is an algorithm through which the change in the two images can be extracted in spatial domain. Mean is the measure of pixel similarity in an image. Subtracting the mean value from each pixel will produce the resultant image which is discriminative. Hence comparing the mean subtracted images will give the difference image.

Mean-Ratio operator=1-min[e1/f1,f1/e1]

Algorithm steps are:

1. Finding the mean of the two images $(\mathrm{m} 1$ and $\mathrm{m} 2)$.

2. Finding the mean ratio $\mathrm{m} 1 / \mathrm{m} 2$ and $\mathrm{m} 2 / \mathrm{m} 1$.

3. Normalize the mean ratio using the equation $[1-\min (\mathrm{m} 1 / \mathrm{m} 2$, $\mathrm{m} 2 / \mathrm{m} 1)]$.

4. Keep these normalized mean as the threshold and make the entire pixel below the threshold as ' 0 ' and above the threshold as ' 1 '. 
Figure. 4.Mean ratio operator

\subsection{Cluster change analysis using log ratio method (LRM):}

The logarithmic operator is characterized by enhancing the lowintensity pixels while weakening the pixels in the areas of high intensity. Therefore, the information of changed regions that is obtained by the log-ratio image may not able to reflect the real changed trends in the maximum extent because of the weakening in the areas of high-intensity pixels.

Log-Ratio operator $=\log 1-\log 2$

Algorithm steps are:

1. Finding the mean of the two images ( $\mathrm{m} 1$ and $\mathrm{m} 2)$.

2. Log ratio is computed using $\log (\mathrm{m} 1)-\log (\mathrm{m} 2)$.

3. Keep this log ratio as the threshold and make the entire pixel below the threshold as ' 0 ' and above the threshold as 1 .

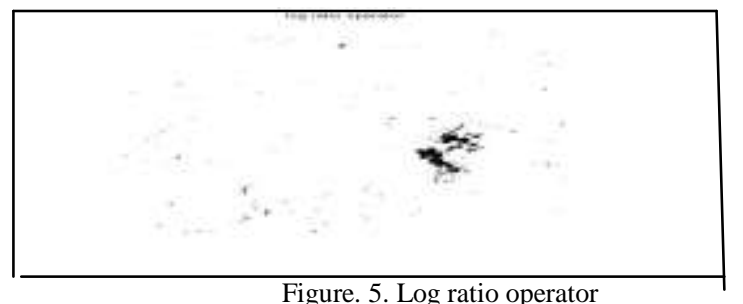

\subsection{Fusing MMRM and LRM using wavelet:}

The implemented wavelet fusion algorithm steps are: Decompose the MMRM image into low-low, low-high, high-low, high-high frequency region using daubechies wavelet. Decompose the LRM image into low-low, low-high, high-low, high-high frequency region using daubechies wavelet.

1. Find the average of every pixels of the low-low region

2. Compare a pixel of the low-high region of the MMRM image with LRM image. Keep the pixel with the lesser value in the resultant image.

3. Compare a pixel of the high-low region of the MMRM image with LRM image. Keep the pixel with the lesser value in the resultant image.

4. Compare a pixel of the high-high region of the MMRM image with LRM image. Keep the pixel with the lesser value in the resultant image.

5. Perform inverse discrete wavelet transform[12] on step 3,4,5,6 images.

www.ijcat.com

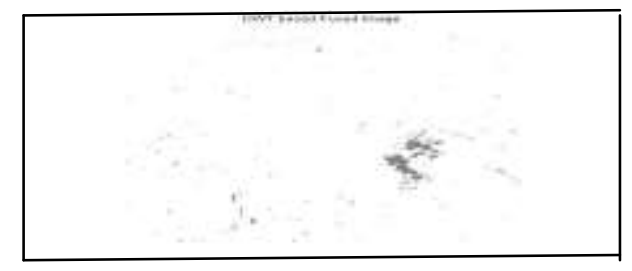

Figure. 6.DWT based fused image

\section{USING FUZZY MODIFIED BY MARKOV RANDOM FIELD}

The Fuzzy c means is well known and popular to distinguish the changed class from the unchanged class in the domain of image segmentation. Here is the algorithm.

\section{FCM ALGORITHM :}

Input : Unlabeled data set consisting of $n$ patterns

Output: Protypes $V$ and fuzzy partition matrix $U$

Step 1: set fusilier $m$ and initialize u randomly

Step 2: Compute each cluster center using (5)

Step 3 : Compute all possible distances $d_{i k}: i=1,2, \ldots c ; k=1,2, . . n$

Step 4 : Update fuzzy partition matrix using (6)

Step 5 : Compute $\Delta=\left\|\left(u_{t}-u_{t-1}\right)\right\|$; t denotes th iteration

Step 6 : Check if $\Delta<\mathcal{E}$, where $\varepsilon$ is a pre defined small positive constant

Step 7 : If above condition is not true goto step 2

Step $8:$ Stop

\section{Description:}

$>$ Choose the number of clusters.

$>$ Assign randomly to each point coefficient for being in the cluster.

$>$ Repeat until the algorithm are converged (that is ,the coefficient change between two iterations is no more than ,the given sensitivity threshold ):

$>$ Compute the centroid of each cluster

$>$ for each point compute its coefficient of being in the clusters

The MRFFCM improves FCM by modifying the membership of each pixel according to the MRF based spatial context. Thecalculated the mean values of the two clusters and the cluster whose center is close to the origin (of the featurespace) is labeled as unchanged and another one as changed. The pixels corresponding to changed one are marked as black (graylevel zero)and unchanged ones are marked as white(graylevel 255) 

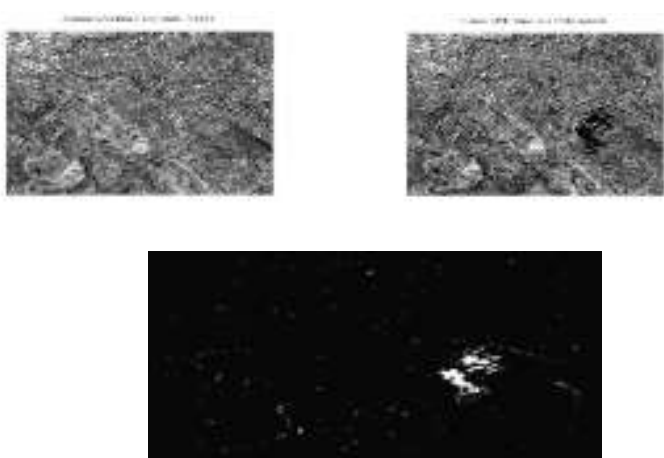

Figure.7.SAR image 1,2 and Segmented image

The figure shows the segmented image after the matching process.

\section{DATASETS DESCRIPTION AND Experimental Settings}

The main by making qualitative visual analysis and quantitative analysis of the experiment results. The preprocess typical corrections have been done before we apply the proposed method .A log ratio and Mean ratio operators are employed to generate difference images. A classical KI method is employed for comparative analysis. A Markov Random Field Fuzzy c-means clustering algorithm[10] (MRFFCM) being a progressive clustering algorithm is employed. By presenting the numerical results on the three datasets we will show the performance of the proposed method. The first data set represents an area near the city of Bern, Switzerland while the second represents city of Ottawa and third represents the region of Yellow river Estuary in China.

\subsection{BERN DATASET:}

The first dataset represents selection $(301 \times 301)$ pixels of two SAR images acquired by the European remote sensing 2 satellite SAR sensor over the city of Bern, Switzerland, in April and May 1999 respectively. Between the two dates, river is flooded in some parts of the cities of Thun and Bern and the airport of Bern entirely. Hence the valley between Bern and Thun was selected as a test site for detecting flooded areas. Here the MRFFCM, shows some changed regions are not detected (appearing as a low value of $\mathrm{FN}$ ).FP of MRFFCM do not exhibit the best, whereas FN, PCC, KC which serves as an overall evaluation are best. It shows that it is less time consuming and time complexity. It does not engender a high time complexity but capable of improving detecting accuracy effectively
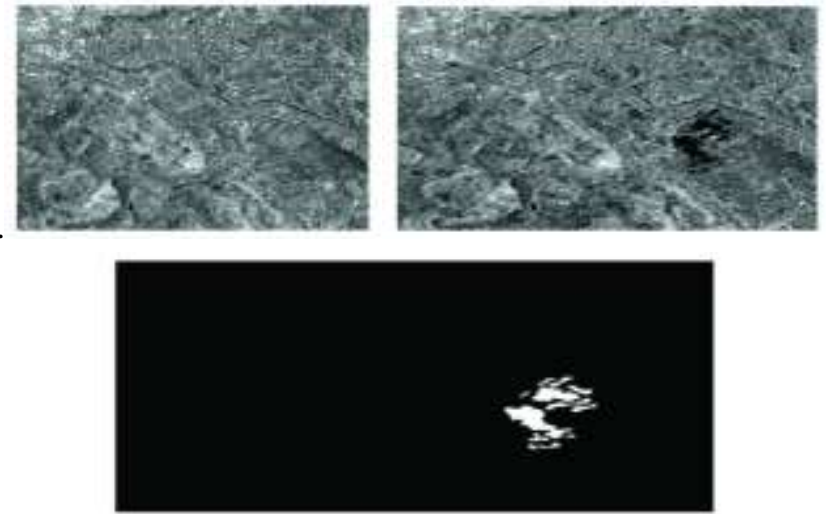

Figure 8.Bern dataset (a) Image acquired in April 1999 (b)Image acquired in May 1999(c) Ground Truth image

\subsection{OTTAWA DATASET}

The second dataset is a section of (290x350) pixels of two SAR images of the city Ottawa acquired by RADARSAT SARsensor. They were provided by defense research and development Canada(DRDC),Ottawa. It shows the image acquired in May 1997 during the summer flooding, shows the image acquired after the summer flooding.
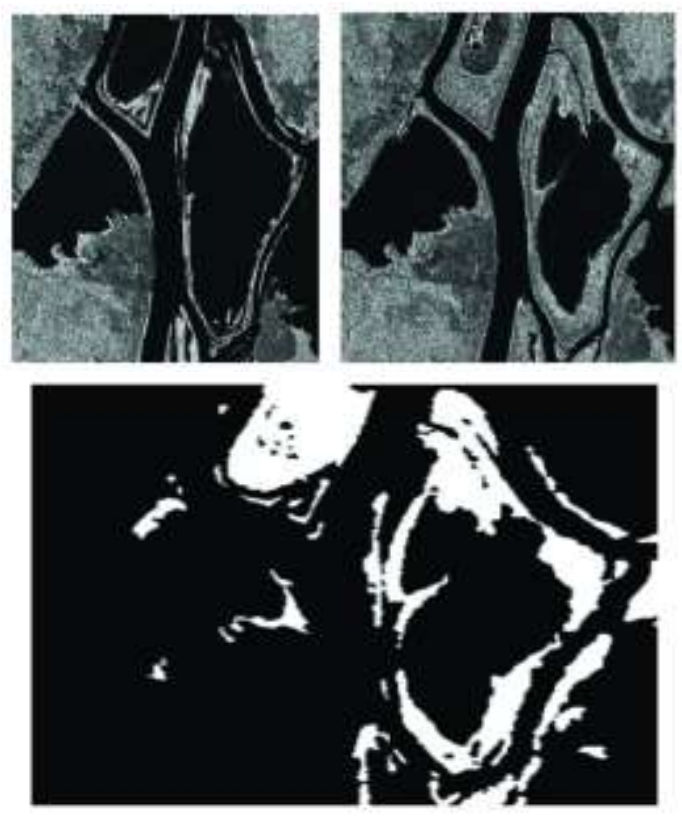

Figure 9. Ottawa dataset (a) Image acquired in May 1997 (b) Image acquired in August 1997(c) Ground Truth image

\subsection{YELLOW RIVER DATASET}

The third dataset comes from two SAR images acquired by RADARSAT- 2 at the region of Yellow river Estuary in China in June 2008 and June 2009 respectively.The 
original SAR images acquired by RADARSAT- 2 are shown and with the size of $7666 \times 7692$. They are too huge to detail the information for our purposes, so only a small typical area of size $306 \times 291$ pixels is selected to compare the change detection results of different approaches. The final map generated by FCM lists those high values of FP. The white spots are eliminated to different degrees by modifying FCM.
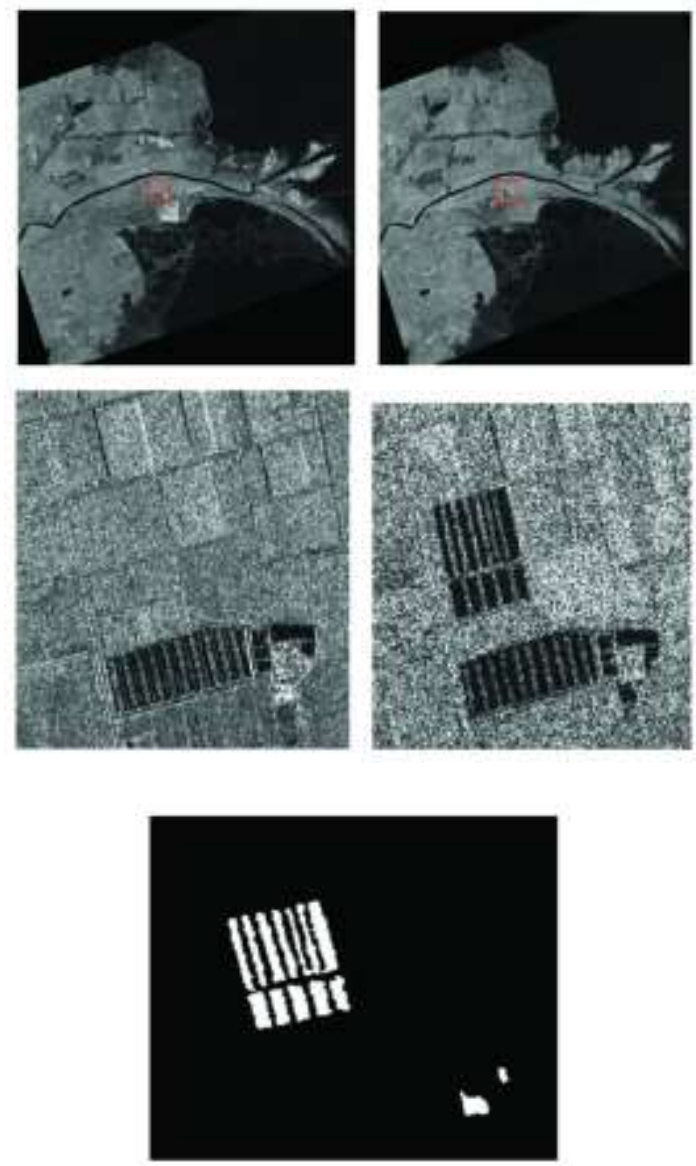

Figure. 10.Yellow river dataset (a) Original Image acquired in 2008 (b) Original Image acquired in 2009 (c) Selected area in (a) (d) Selected area in (b) .(c) Ground Truth image of the selected area.

The following measures are computed for change detection results.True positive (TP) is the number of pixels that are detected as the changed area both in the reference image and in the change detection result. True negative (TN) is the number of pixels that are detected as the unchanged area both in the reference image and in the change detection result.False negative (FN) is the number of pixels that are detected as changed area in the reference image and as unchanged area in the change detection result. False positive (FP) is number of pixels that are detected as unchanged area in the reference image and as changed area in the change detection result.Overall error $(\mathrm{OE})$ is the total number of decision errorswhich equals the sum of FN and FP. The percentage correct classification (PCC) is calculated as follows:

www.ijcat.com

$$
\begin{gathered}
\mathrm{PCC}=\mathrm{TP}+\mathrm{TN} \\
\mathrm{TP}+\mathrm{TN}+\mathrm{FP}+\mathrm{FN} .
\end{gathered}
$$

\section{Table 1. PCC calculation}

\begin{tabular}{|r|r|r|r|r|r|r|}
\hline Datasets & $\boldsymbol{F P}$ & $\boldsymbol{F N}$ & $\boldsymbol{O E}$ & $\boldsymbol{P C C}$ & $\boldsymbol{K C}$ & $\boldsymbol{T / s}$ \\
\hline BERN & 2615 & 6 & 2621 & 0.99 & 1 & 56.8 \\
\hline OTTAWA & 2248 & 113 & 2361 & 0.99099 & 1 & 71.0 \\
\hline $\begin{array}{r}\text { YELLOW } \\
\text { RIVER }\end{array}$ & 2617 & 4 & 2621 & 0.99 & 1 & 66.1 \\
\hline
\end{tabular}

In addition, Kappa statistic taking into account of commissionand omission errors is employed for evaluating theperformance comparison of the methods. Kappa statisticscalculated as follows:

$$
\text { Kappa }=\text { PCC }-\mathrm{PRE} / 1-\mathrm{PRE}
$$

where

$$
\begin{gathered}
\mathrm{PRE}=((\mathrm{TP}+\mathrm{FP})(\mathrm{TP}+\mathrm{FN})+(\mathrm{FN}+\mathrm{TN})(\mathrm{TN}+\mathrm{FP})) /(\mathrm{TP}+\mathrm{TN}+\mathrm{FP} \\
+\mathrm{TN}) 2 .
\end{gathered}
$$

If the change detection result and the reference image are in full agreement, the Kappa value is 1 . If there is no agreement between the change detection result and the reference image, the Kappa value is 0 .

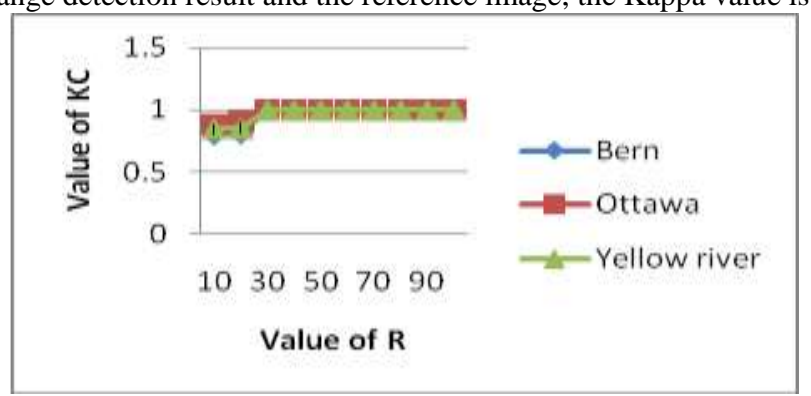

Figure.11. Values of the Evaluation criteria of the three datasets

The testing curve of the parameter $\mathrm{R}$ on the three datasets is employed.

\section{CONCLUSION}

The analysis of multitemporal SAR images has been done. This approach is based on the universal utilized FCM algorithm and the MRF model. After the difference image has been generated through the log-ratio operator, the MRF method is noted in the procedure of FCM algorithm. The energy function is altered by utilizing not only the membership but also the number of same class of neighborhood pixels. Thus we are able to decide whether the central pixels are in the homogenous region or in the heterogeneous region.

The new approach does not consider the use of any prior knowledge about the scene but it considers only the use of gray-level intensity. So it is an unsupervised approach. The main advantages of change detection approach is reducing the speckle noise, making the computations are simpler and it has low complexity. To compute the SAR images by the technique of image fusion, the image is normally converted from discrete waveform to Stationary wavelet transform (SWT).This technique is achieved and efficiency is also increased.

\section{REFERENCES}

[1] M. Gong, Z. Zhou, and J. Ma, "Change detection in synthetic aperture radar images based on image fusion and fuzzy 
clustering," IEEE Trans.Image Process., vol. 21, no. 4, pp. 21412151, Apr. 2012.

[2]S. Krinidis and V. Chatzis, "A robust fuzzy local information C-meansclustering algorithm," IEEE Trans. Image Process., vol. 19, no. 5,pp. 1328-1337, May 2010.

[3]X. Zhang, H. Li, and C. Qi, "Spatially constrained fuzzyclustering-basedsensor placement for spatiotemporal fuzzy-control system," IEEE Trans.Fuzzy Syst., vol. 18, no. 5, pp. 946-957, Oct. 2010.

[4]D. M. Tsai and S. C. Lai, "Independent component analysisbased background subtraction for indoor surveillance," IEEE Trans. Image Process.,vol. 18, no. 1, pp. 158-167, Jan. 2009.

[5]A. Robin, L. Moisan, and S. Hegarat-Mascle, "Anacontrario approach robust to registration noise for change detection in VHR multispectral images," IEEE Trans. Image Process., vol. 19, no. 7 , pp. $1877-1889$, Jul.2010.

[6]S. S. Ho and H.Wechsler, "Amartingale framework for detecting changes for subpixel change detection in satellite imagery," IEEE Trans. Pattern Anal. Mach. Intell., vol. 32, no. 11, pp. 19771993, Nov. 2010.

[7]S. Marchesi, F. Bovolo, and L. Bruzzone, "A contextsensitive technique in data streams by testing exchangeability," IEEE Trans. Pattern Anal.Mach.Intell., vol. 32, no. 12, pp. 2113-2127, Dec. 2010.

[8]H. C. Huang, Y. Y. Chuang, and C. S. Chen, "Multiple kernel fuzzy clustering,"IEEE Trans. Fuzzy Syst., vol. 20, no. 1, pp. 120-134, Feb. 2012.

[9]F. Chatelain, J.-Y. Tourneret, and J. Inglada, "Change detection in multisensory SAR images using bivariate gamma distributions," IEEE Trans.Image Process., vol. 17, no. 3, pp. 249-258, Mar. 2008.

[10]W. Cai, S. Chen, and D. Zhang, "Fast and robust fuzzy C-means clustering algorithms incorporating local information for image segmentation," Pattern Recog., vol. 40, no. 3, pp. 825-838, Mar. 2007.

[11]Ashish Ghosh ,Niladri Shekhar Mishra , Susmita Ghosh , "Fuzzy clustering algorithms for unsupervised change detection in remote sensing images", journal paper in information sciences .

[12]Ferdinando Di Martino, Vincenzo Loia , Salvatore Sessa ,'Direct and Inverse Fuzzy Transforms for Coding/Decoding Color Images in YUV Space", Journal of Uncertain Systems Vol.3, No.1, pp.11-30, 2009.

[13] Nguyen XuanVinh ,Julien Epps and James Bailey, "Information Theoretic Measures for Clusterings Comparison:Variants, Properties Normalization and Correction for Chance",Journal of Machine Learning Research 11 (2010)

Ms.S.Venkata Lakshmi is currently working as a Assistant Professor Grade 1, Computer Science and Engineering, at Panimalar Institute of Technology, Poonamalle, Chennai 600062.She has completed her graduate in M.S.University and Post graduate in DR.MGR university where she is currently working toward the $\mathrm{Ph} . \mathrm{D}$ degree. She has published research papers in International, National conference proceedings. Her research interest includes image processing and medical imaging.

Mr.K.Sathyamoorthy is currently working as a Assistant Professor, Computer Science and Engineering, at Panimalar Institute of Technology, Poonamalle, Chennai 600062. He has completed his graduate in Anna University and Post graduate in Sathyabama University. His researchinterest includes image processing and soft computing.
Mr. T.K Senthilkumar is currently working as a Assistant Professor, Electronic and Communication Engineering at Rajalakshmi Institute of Technology, Poonamalle, Chennai 600124.He has completed his graduate and Post graduate in Anna University. He is currently working toward the Ph.D degree. He has published research papers in International, National journals. His research interests includeimage / signal processing and VLSI. 